\title{
Recombinant Betacellulin
}

National Cancer Institute

\section{Source}

National Cancer Institute. Recombinant Betacellulin. NCI Thesaurus. Code C1483.

A recombinant form of the endogenous Betacellulin, a member of the Epidermal Growth Factor family that is expressed primarily in the pancreas and induces differentiation of a pancreatic acinar cell line. 